\title{
Comparison of Efficacy of Oral Bromelain and Serratiopeptidase for the Control of Postoperative Sequelae Following Third Molar Surgery - A Comparative Study
}

\author{
Subhashini Ramasubbu' ${ }^{1}$, Abdul Wahab P. U. ${ }^{2}$ \\ 1,2 Department of Oral and Maxillofacial Surgery, Saveetha Dental College and Hospitals, \\ SIMATS, Chennai, Tamil Nadu, India.
}

\section{ABSTRACT}

\section{BACKGROUND}

Surgical removal of impacted third molar is widely carried out in general dental practice and is usually associated with postoperative inflammatory sequelae like pain, swelling, and trismus. The objective of the study was to assess and compare the efficacy of oral bromelain and Serratiopeptidase for the control of post-operative sequelae following surgical removal of impacted third molar.

\section{METHODS}

A randomized, comparative clinical study was conducted on sixty patients who were undergoing impacted third molar surgery, and were allocated into two groups each comprising 30 patients. Conventional drugs included Cap amoxicillin $500 \mathrm{mg}$ bid, Tab. metronidazole $200 \mathrm{mg}$ tid, and Tab. diclofenac sodium $50 \mathrm{mg}$ bid that were given to both the groups. Group I was treated with Tab. bromelain $200 \mathrm{mg}$ tid along with conventional treatment for 5 days. Group II received a combination of conventional treatment and Tab. Serratiopeptidase $10 \mathrm{mg}$ bid for 5 days. The parameters such as measurement of pain, facial width and trismus were analysed on postoperative days 1,2 and 7.

\section{RESULTS}

The study comprised of totally 60 otherwise healthy patients. The group of patients who received bromelain along with diclofenac sodium seemed to be effective in controlling post-operative sequelae like pain $(\mathrm{P}<0.05)$, swelling $(\mathrm{P}<0.05)$ but not trismus $(\mathrm{P}<0.05)$ when compared to the group who received Serratiopeptidase with diclofenac sodium.

\section{CONCLUSIONS}

The intensified anti-inflammatory action of bromelain, was possibly by inhibiting the generation of bradykinin and negative action on the prostaglandin pathway. The present study assessed the clinical effect of bromelain and serratiopeptidase on pain, facial swelling and trismus.

\section{KEY WORDS}

Bromelain, Oral Bromelain, Serratiopeptidase, Proteolytic Enzyme, Third Molar Surgery

\section{Corresponding Author:} Dr. Subhashini Ramasubbu, Dept. of Oral and Maxillofacial Surgery, Saveetha Dental College and Hospitals, SIMATS, Chennai, Tamil Nadu India. E-mail:meetsubha07@gmail.com

\section{DOI: $10.14260 /$ jemds $/ 2021 / 506$}

\section{How to Cite This Article:}

Ramasubbu S, Wahab PUA. Comparison of efficacy of oral bromelain and serratiopeptidase for the control of postoperative sequelae following third molar surgery - a comparative study. J Evolution Med Dent Sci 2021;10(31):24762480, DOI: $10.14260 /$ jemds/2021/506

Submission 25-03-2021,

Peer Review 29-05-2021,

Acceptance 05-06-2021,

Published 02-08-2021.

Copyright (C) 2021 Karishma Anurag Kapur et al. This is an open access article distributed under Creative Commons Attribution License [Attribution 4.0 International (CC BY 4.0)] 


\section{BACKGROUND}

Impacted third molar removal is usually a chair side procedure performed by oral and maxillofacial surgeons and it is the most common procedure to be performed in dental office..$^{1,2}$ Like any other surgical procedures third molar removal is also associated with several immediate and late complications. Most common complications include pain, swelling, restricted mouth opening, paraesthesia etc. The unfavourable effects of the third molar removal on the quality of life of patients ${ }^{3,4}$ has been recorded to show a threefold increase in patients who experience pain, swelling and trismus alone or in combination when compared to those who were asymptomatic.5,6 So there is a need for pain control, reduction in swelling without restriction of mouth opening in patients undergoing wisdom tooth removal. ${ }^{7}$ Prostaglandins and Prostacyclins are responsible for pain and oedema in inflammatory cascade. The mechanism of action of nonsteroidal anti-inflammatory drugs is to prevent prostaglandin and prostacyclin production thereby reducing the pain and swelling due to inflammation. ${ }^{8}$ The use of oral systemic enzyme therapy like bromelain, serratiopeptidase and trypsin provided added benefits for controlling the inflammatory sequelae following impacted third molar removal.9,10

Bromelain is a proteolytic enzyme present in tissues of the plant family bromeliaceae, of which pineapple, is the best known. Bromelain possesses many properties which include anti-inflammatory, ${ }^{11}$ antiemetic, anti-tumour, and immunomodulatory effects. The mechanism of the antiinflammatory activity is, it inhibits bradykinin generation, proteolytic degradation of circulating immune complexes and interference with the arachidonic acid metabolism.12,13 It promotes the absorption of antibiotic drugs topically in the skin debridement of burns. Bromelain has been used as an additive agent for anticancer therapy. ${ }^{14,5}$ Serratiopeptidase is also a proteolytic enzyme obtained from silkworms. Serratia E15 is a microorganism which lives in the gut producing the enzyme. This causes proteolysis of all non-vital tissues including blood clots, tissue plaques and cellular debris and reduces the inflammatory response. The anti-inflammatory activity of serratiopeptidase helps in resolution of postpartum hematomas and breast enlargements, it reduces internal tissue oedema caused at postoperative handling. Serratiopeptidase does not inhibit prostaglandins and so it is safe to the GI system. ${ }^{16}$

This study is based on the proposed hypothesis that bromelain can be more efficient in terms of reducing pain and swelling. Co-administration of oral systemic enzyme and NSAIDs may produce pronounced anti-inflammatory effects without side effects. ${ }^{9,17}$ The rationale of this study was to assess and compare the efficacy of bromelain and serratiopeptidase in controlling the post-operative inflammatory sequelae following wisdom tooth removal when given with NSAIDs.

\section{METHODS}

A randomized, clinical study was conducted on a total of 60 patients requiring surgical removal of impacted third molar teeth. Sample size was calculated using G power based on the mean and SD of a study done by NK Tiwari et al. ${ }^{18}$ The recruitment period of the study was 3 months (November 2019 - February 2020) at Saveetha Dental College and Hospitals, SIMATS, Chennai. The study protocols were approved by the Institutional ethical committee. Consent was taken prior to the surgery and the procedures were adhered to the ethical guidelines of the Declaration of Helsinki.

The inclusion criteria were patients belonging to age group of 20 - 40 years, both genders, patients willing to give informed consent, patients willing to adhere to the study protocol, patients with no history of oral surgical interventions during the past 3 weeks, patients with free of pain and other inflammatory symptoms that included swelling and decreased mouth opening at the time of surgery.

Exclusion criteria were pregnant and lactating women, patients with cardiac, renal or hepatic disease, and patients with previous or present gastric ulcers, known hypersensitivities, allergies, or idiosyncratic reactions to any study medications, patients who had taken anti-inflammatory drugs within 2 days before surgery, patients not willing to give informed consent.

Sixty patients who fulfilled inclusion and exclusion criteria were included in the study and they were randomly allocated into two groups following simple randomisation. SNOSE method was followed and each group comprising 30 patients received the respective study medication orally for 5 days. Conventional drugs include Cap amoxicillin $500 \mathrm{mg}$ bid, Tab. metronidazole $200 \mathrm{mg}$ tid, and Tab. diclofenac sodium $50 \mathrm{mg}$ bid were given to both the groups. Group I was treated with Tab. bromelain $200 \mathrm{mg}$ tid along with conventional treatment for 5 days. Group II received a combination of conventional treatment and Tab. serratiopeptidase $10 \mathrm{mg}$ BD for 5 days.

The first dose of the study medication was administered to the respective group of patients 30 minutes prior to the surgery. The proper postoperative care instructions and medication directions were given to all the participants. The following study parameters were recorded on $1 \mathrm{st}$, $2 \mathrm{nd}$ and 7 th postoperative days.

\section{Pain Measurement}

Preoperative pain was assessed using a Visual Analogue Scale (VAS). Accordingly, pain was recorded as: 0 - No pain, 1 - 3 Mild pain, 3 - 5 Less moderate pain, 5 - 7 Intense moderate pain and 7 - 9 Severe pain, 10 - Worst pain possible. For each patient, the appropriate score was recorded by the investigator at preoperative, postoperative days 1, 2 and 7. Before leaving the clinic, the investigator ensured that all patients were thoroughly instructed on how and when to take medications.

\section{Measurement of Facial Width}

As there is no published method that satisfies all criteria to assess facial swelling, we used a measuring tape to measure facial width and swelling in one-dimension only. The reference points were the tip of the tragus of right and left ears, with the gonium in between. The measurements were carried out (in $\mathrm{mm}$ ) just before the surgery and at 1st, 2 nd and 7 th postoperative days. 


\section{Measurement of Mouth Opening}

A Caliper was used to measure maximum interincisal mouthopening at the commencement of the procedure with maximum mouth opening. The measurements were made in millimetres $(\mathrm{mm})$. The measurement was carried out just before the surgery and at 1 st, 2 nd and 7 th postoperative days. No Confounders in this study.

\section{Methodology Flow Chart}

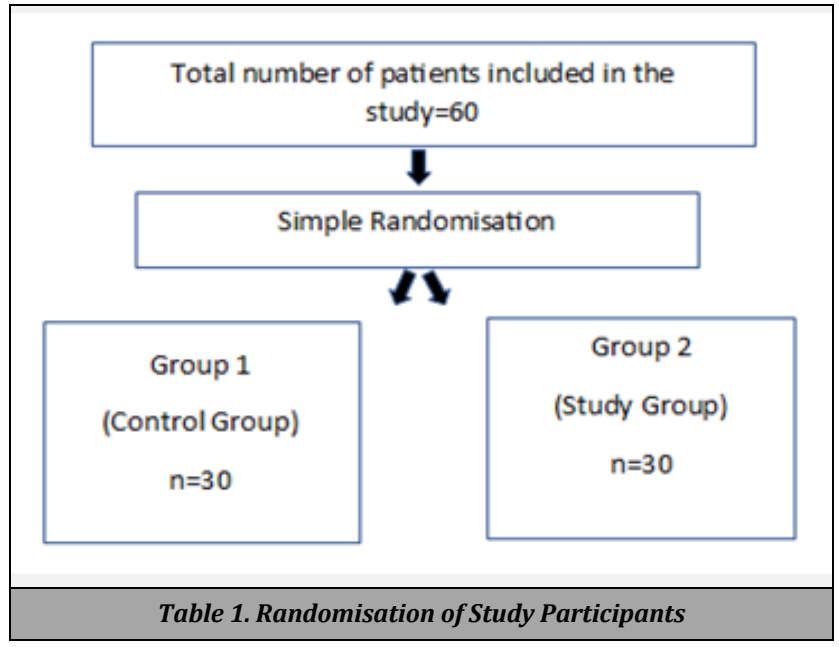

\section{Statistical Analysis}

Statistical analyses were performed using SPSS for Windows, version 20 (IBM Corp, Armonk, NY. Student t test was used for Visual Analogue Scale, interincisal opening and facial swelling. The level of significance was set at $\mathrm{P}<0.05$.

\section{RESULTS}

A total of 60 patients distributed into group I $(\mathrm{N}=30)$, II $(\mathrm{N}=$ 30) who completed the study were included in the analysis. The anti-inflammatory effects of bromelain and serratiopeptidase as an add on therapy with conventional treatment (diclofenac) following surgical removal of impacted mandibular third molar were compared.

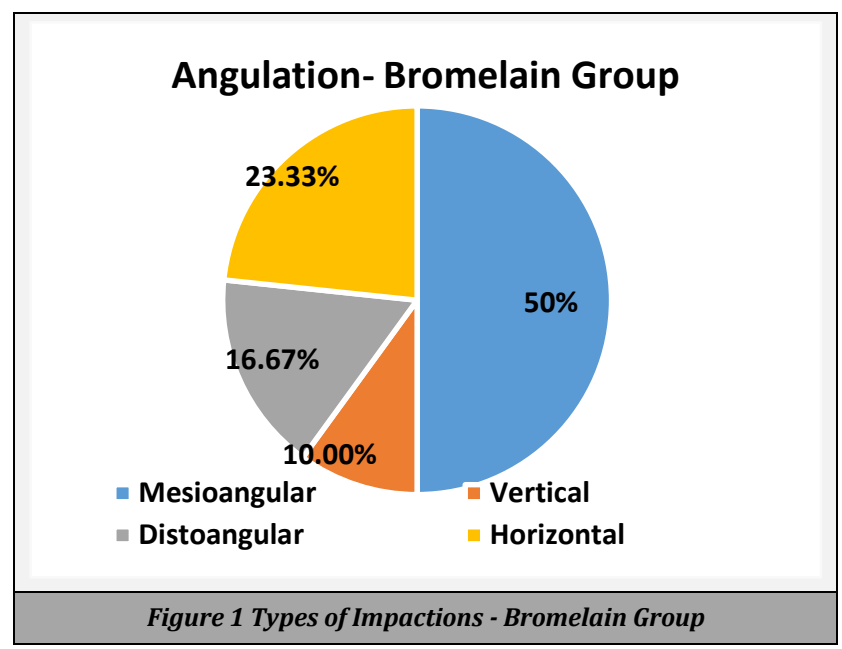

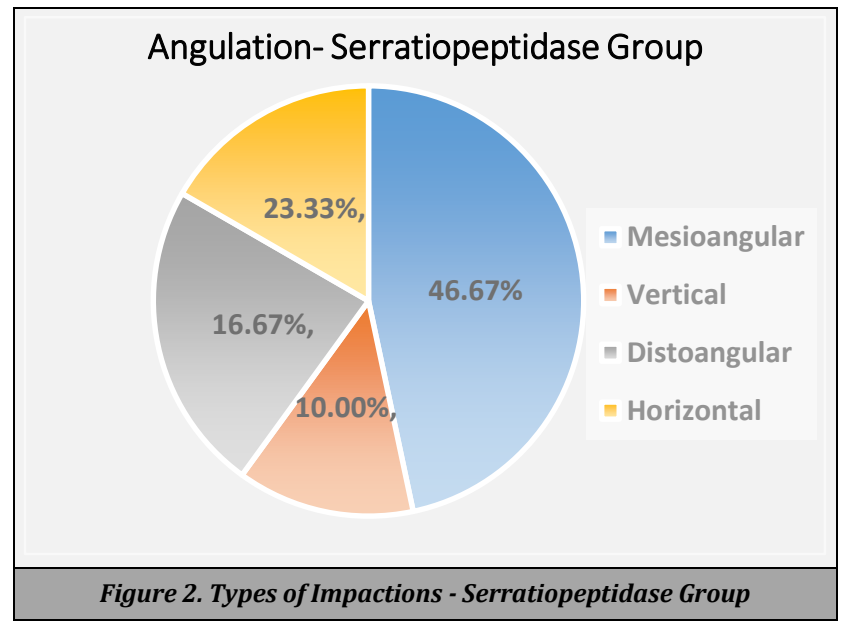
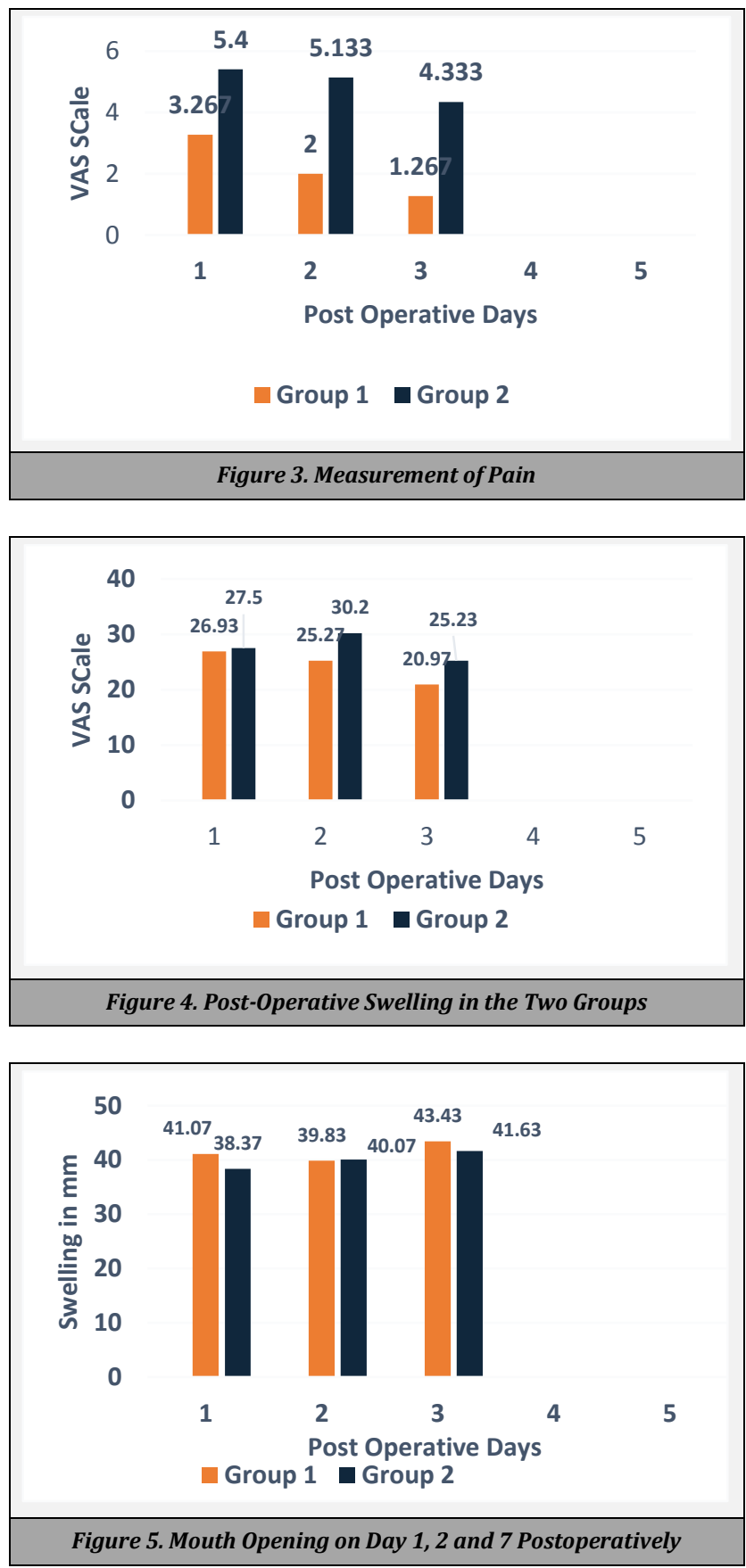

Figure 3 represents comparison of mean post -operative pain on day 1,2 and 7 and the bromelain group has 
considerably less pain compared to the serratiopeptidase group. Figure 4 represents comparison of Mean of postoperative swelling on day 1, 2 and 7, bromelain decreases postoperative swelling compared to serratiopeptidase group. Figure 5 represents comparison of mean of mouth opening on postoperative day 1, 2 and 7 and there was no significant improvement in mouth opening in both the groups.

\section{DISCUSSION}

Prostaglandins (PG) play a major role in initiation of inflammation. ${ }^{19}$ The mechanism of action of NSAIDs is to inhibit COX pathway and thereby lowers the production of prostaglandins. ${ }^{20}$ Preoperative administration of nonsteroidal anti-inflammatory drugs is reported to be efficient in minimising pain and other sequelae of inflammation. ${ }^{21}$ Like COX pathway NSAIDs are known to block arachidonic acid pathway also.

The rationale behind administration of NSAID before surgery is, the drug is widely absorbed and distributed in the tissues before any surgical insult happens. So the prostaglandin synthesis is also reduced before initiating any trauma $^{22}$ and thereby the inflammatory sequelae. If the inflammatory sequelae are prevented before the trauma, then there will be reduction of pain and swelling. Many drugs are shown to be effective in controlling the above said sequelae. One among those is diclofenac sodium which has both analgesic and anti-inflammatory properties. ${ }^{23}$

To reduce the postoperative inflammation, we can administer oral systemic enzymes. Bromelain / serratiopeptidase have the ability to interfere with inflammation cascade and, thus reduces pain and swelling. ${ }^{24,25}$ Studies also stated that administration of bromelain / serratiopeptidase lowers the bradykinin production. 16

The enhanced anti-inflammatory action of bromelain, possibly by inhibiting the generation of bradykinin is negative action on the prostaglandin pathway. As known, pain and enhanced vascular permeability are caused by kinins namely bradykinin and kallikrein. Both kinins may act alone as well as together. Due to its anti-inflammatory effects, the administration of oral systemic enzymes reduces inflammatory cascades like pain and swelling. So, when given with NSAIDs these enzymes may lower the kinin levels.

This study analysed the clinical efficacy of bromelain and serratiopeptidase on pain, facial swelling, and trismus. There is a significant difference in terms of pain in bromelain group when compared to serratiopeptidase group $(\mathrm{P}<0.05)$. This is in accordance with previous literatures. ${ }^{26-28}$

Post-operative facial oedema is difficult to quantify accurately. Many methods are there to objectively measure the swelling. In this study we have measured from tip of the tragus to gonion to the contralateral tragus. A single measurement was taken. The bromelain group showed reduction in swelling post operatively. This result shows that bromelain effectively decreases postoperative facial swelling than serratiopeptidase. This is in accordance with previous literatures. ${ }^{29}$

The mean pain scores on day 1 in group I were significantly lower than that of group II. This result shows that addition of bromelain enhances the control of postoperative pain on day 1.

The mean pain score on day two in group I was also significantly lower than that of group II. This result shows that patients who received bromelain and diclofenac have greater control of postoperative pain than other groups. The result is in accordance with previous literatures. However, there is no significant reduction in trismus in both the groups $(\mathrm{P}>0.05)$.

\section{CONCLUSIONS}

The present study concludes that anti-inflammatory effects of bromelain on postoperative pain, facial swelling were enhanced compared to the serratiopeptidase group following impacted third molar removal. However, there was no significant improvement in trismus (mouth opening) between bromelain and serratiopeptidase group. Thus this study helped us to understand the importance of oral proteolytic enzymes and proved to have a beneficial role in coadministration with conventional therapy in the management of pain and inflammation.

Data sharing statement provided by the authors is available with the full text of this article at jemds.com.

Financial or other competing interests: None.

Disclosure forms provided by the authors are available with the full text of this article at jemds.com.

\section{REFERENCES}

[1] Thomas D, Walker R, Smith A, et al. The provision of oral surgery services in England and Wales 1984-1991. Br Dent J 1994;176(6):215-9.

[2] Van Der Westhuijzen AJ, Roelofse JA, Grotepass FW, et al. Randomized double-blind comparison of tiaprofenic acid and diclofenac sodium after third molar surgery. Oral Surg Oral Med Oral Pathol 1994;78(5):557-66.

[3] Antila H, Lehtinen R, Heinaro I, et al. Successful pain management by Finnish oral surgeons. A clinical followup study. Oral Surg Oral Med Oral Pathol 1992;74(1):1923.

[4] McGrath C, Comfort MB, Lo ECM, et al. Changes in life quality following third molar surgery-the immediate postoperative period. Br Dent J 2003;194(5):265-8.

[5] Ruta DA, Bissias E, Ogston S, et al. Assessing health outcomes after extraction of third molars: the postoperative symptom severity (PoSSe) scale. Br J Oral Maxillofac Surg 2000;38(5):480-7.

[6] Slade GD, Foy SP, Shugars DA, et al. The impact of third molar symptoms, pain and swelling on oral health-related quality of life. J Oral Maxillofac Surg 2004;62(9):1118-24.

[7] Ogden GR. Third molar surgery and postoperative pain relief. Br Dent J 2003;194(5):261.

[8] Moore PA, Brar P, Smiga ER, et al. Preemptive rofecoxib and dexamethasone for prevention of pain and trismus following third molar surgery. Oral Surg Oral Med Oral Pathol Oral Radiol Endodontol 2005;99(2):E1-7.

[9] De Souza GM, Fernandes IA, Dos Santos CRR, et al. Is bromelain effective in controlling the inflammatory 
parameters of pain, edema and trismus after lower third molar surgery? A systematic review and meta-analysis. Phytother Res 2019;33(3):473-81.

[10] Murugesan K, Sreekumar K, Sabapathy B. Comparison of the roles of serratiopeptidase and dexamethasone in the control of inflammation and trismus following impacted third molar surgery. Indian J Dent Res 2012;23(6):709. 13.

[11] Müller S, März R, Schmolz M, et al. Placebo-controlled randomized clinical trial on the immunomodulating activities of low-and high-dose bromelain after oral administration-new evidence on the antiinflammatory mode of action of bromelain. Phytother Res 2013;27(2):199-204.

[12] Bernela M, Ahuja M, Thakur R. Enhancement of antiinflammatory activity of bromelain by its encapsulation in katira gum nanoparticles. Carbohydr Polym 2016;143:18-24.

[13] Errasti M, Caffini N, Pelzer L, et al. Anti-inflammatory activity of bromelia hieronymi: comparison with bromelain. Planta Medica 2013;79(3-4):207-13.

[14] Amini A, Masoumi-Moghaddam S, Ehteda A, et al. Potentiation of chemotherapeutics by bromelain and $\mathrm{N}$ acetylcysteine: sequential and combination therapy of gastrointestinal cancer cells. Am J Cancer Res 2016;6(2):350-69.

[15] Mohamad NE, Abu N, Yeap SK, et al. Bromelain enhances the anti-tumor effects of cisplatin on 4T1 breast tumor model in vivo. Integr Cancer Ther 2019;18:1534735419880258.

[16] Sri Neeraja P. Role of Serratiopeptidase in Enzyme Therapy. International Journal of Science and Research 2017;6(7):758-9.

[17] Liu S, Zhao H, Wang Y, et al. Oral Bromelain for the control of facial swelling, trismus and pain after mandibular third molar surgery: a systematic review and meta-analysis. J Oral Maxillofac Surg 2019;77(8):1566-74.

[18] Tewari NK, Kundan K, Tiwari S, et al. Evaluation of effect of multiple sutures versus suture less technique in $3^{\text {rd }}$ molar extraction followed by inflammatory complication. Int J Res Health Allied Sci 2019;5(6):17-9.

[19] Tyers MB, Haywood H. Effects of prostaglandins on peripheral nociceptors in acute inflammation. Agents Actions Suppl 1979;6:65-78.
[20] Vane JR. Introduction: mechanism of action of NSAIDs. Br J Rheumatol 1996;35(Suppl 1):1-3.

[21] Wang C, Fu H, Wang J, et al. Preemptive analgesia using selective cyclooxygenase-2 inhibitors alleviates postoperative pain in patients undergoing total knee arthroplasty: a protocol for PRISMA guided meta-analysis of randomized controlled trials. Medicine (Balimore) 2021;100(7):e24512.

[22] Harvey W. Inflammation, cytokines and prostaglandins. In: Prostaglandins in Bone Resorption. CRC Press 2020:57-72.

[23] Gorecki P, Rainsford KD, Taneja P, et al. Submucosal diclofenac for acute postoperative pain in third molar surgery: a randomized, controlled clinical trial. J Dent Res 2018;97(4):381-7.

[24] Desjardins P, Alvarado F, Gil M, et al. Efficacy and safety of two fixed-dose combinations of tramadol hydrochloride and diclofenac sodium in postoperative dental pain. Pain Med 2020;21(10):2447-57.

[25] De A C Almeida R, De Sousa Lima FCM, Do E Vasconcelos BC. Is bromelain an effective drug for the control of pain and inflammation associated with impacted third molar surgery? Systematic review and meta-analysis. Int J Oral Maxillofac Surg 2019;48(5):651-8.

[26] Soheilifar S, Bidgoli M, Hooshyarfard A, et al. Effect of oral bromelain on wound healing, pain and bleeding at donor site following free gingival grafting: a clinical trial. J Dent (Tehran) 2018;15(5):309-16.

[27] Ghensi P, Cucchi A, Creminelli L, et al. Effect of oral administration of bromelain on postoperative discomfort after third molar surgery. J Craniofac Surg 2017;28(2):e191-7.

[28] Mendes MLT, Do Nascimento-Júnior EM, Reinheimer DM, et al. Efficacy of proteolytic enzyme bromelain on health outcomes after third molar surgery. Systematic review and meta-analysis of randomized clinical trials. Med Oral Patol Oral Cir Bucal 2019;24(1):e61-9.

[29] Wala LJ, Choudhary A, Reddy BC. Clinical evaluation of anti-inflammatory properties of combination of bromelain, trypsin and rutoside with combination of ibuprofen, trypsin and chymotrypsin following third molar extraction-a comparative study. Journal of Medical Science and Clinical Research 2020;8(2):464-8. 